Ann. Zootech., I968, 17 (I), 23-30.

\title{
LE SEVRAGE PRÉCOCE DES LAPEREAUX ET LA REPRODUCTION DES LAPINES.
}

\author{
M. PRUD'HON, L. BEL \\ Laboratoire de Zootechnie, \\ Centre de Recherches agronomiques du Midi, \\ École nationale supérieure agronomique, 34 - Montpellier \\ Institut national de la Recherche agronomique
}

SOMMAIRE

Nous décrivons dans cette note une méthode de sevrage des lapereaux à l'âge de deux semaines à l'aide d'une alimentation sèche à base de poudre de lait et de granulés.

Les vitesses de croissance et les taux de mortalité, comparés à ceux des témoins allaités jusqu'à sept semaines, n'en diffèrent pas de façon significative.

Nous donnons également les résultats obtenus par saillie de lapines le jour de la mise bas. Ni le taux de gestation, ni la taille des portées ne diffèrent significativement de ceux des femelles soumises à un rythme traditionnel de reproduction avec sevrage à sept semaines.

La possibilité d'accroître la productivité des lapines par l'action conjuguée du sevrage précoce des lapereaux et l'accélération du rythme de reproduction est mise en valeur.

\section{INTRODUC'TION}

Le sevrage des lapins est pratiqué traditionnellement entre six et huit semaines, au moment où la production laitière des femelles diminue rapidement et où celles-ci commencent à reconstituer leurs réserves. Dans ces conditions, le nombre de portées produites annuellement ne dépasse généralement pas quatre à cinq par mère. Dans le but d'accélérer le rythme de reproduction des lapines, certains éleveurs ont tenté, avec succès, de sevrer à quatre semaines SoUbIEI,LE (Ig66) ou même trois semaines DON GuTHRIf, (Ig66). Par ailleurs, Zarrow et al. (I965) ont constaté que la consommation d'aliments solides par de jeunes lapins allaités par leur mère pouvait débuter spontanément le seizième jour suivant la mise bas et qu'elle était accompagnée d'une accélération immédiate de la croissance. De plus, nous avons remarqué (PRUD'Hov I967), que l'indice de consommation des portées s'abaissait très sensiblement lorsque les petits commençaient à manger du granulé. 
Dans ces conditions il nous a semblé intéressant:

- d'essayer de sevrer des lapins précocement, à partir de quatorze jours ou même avant,

- de soumettre les lapines dont les petits seront sevrés précocement, à un accouplement le jour même de la mise bas.

Nous décrivons ici la technique de sevrage utilisée et les premiers résultats de ces essais.

\section{MATÉRIEL D'ÉTUDE E'T TECHNIQUES DE SEVRAGE}

\section{Matériel d'étude}

Les essais de sevrage précoce ont porté sur 178 lapereaux de deux semaines appartenant à 24 portées issues de 16 lapines. L'alimentation des 16 premières portées sevrées a été contrôlée. Le lot témoin était composé de 107 lapereaux appartenant à 17 portées issues de 17 lapines.

Les lapines utilisées appartiennent à des souches communes, de format moyen $(4 \mathrm{~kg}$ à l'âge adulte), élevées depuis 1960 au laboratoire. Les femelles sont placées en cages individuelles sur grillage, elles reçoivent à volonté un granulé dont la composition figure au tableau I, de l'eau, ainsi que du foin en début de gestation, elles sont soumises à un rythme lumineux de it $\mathrm{h}$ de lumière, Io h d'obscurité et à des conditions d'élevage décrites antérieurement (PruD'HON, I967).

TABI EAU I

Composition des aliments

\begin{tabular}{|c|c|c|}
\hline & Lait & Granulé (1) \\
\hline \multirow[t]{2}{*}{ Matière sèche } & 94,8 & 90,3 \\
\hline & P. 100 de la MS & P. 100 de la $\mathrm{KS}$ \\
\hline Matières cellulosiques ........ & $\ldots$ & $1 \div$ \\
\hline Matières grasses............ & 27,4 & 3,6 \\
\hline Matières azotées .......... & 27,4 & $2,2,7$ \\
\hline Extractif non azoté ....... & 38,8 & 50,1 \\
\hline Matières minérales ......... & 6,4 & 9,6 \\
\hline
\end{tabular}

Le granulé contiendrait, en outre, $11000 \mathrm{UI}$ de vitamine $\mathrm{A}$ jar $\mathrm{kg}$ 6000 LI de vitamine $\mathrm{D}_{4}$ jar $\mathrm{kg}$ $0,05 \mathrm{~g}$ de Furoxone par $\mathrm{kg}$.

(1) Gramulé « lapines mères lapereaux ", Établissements Martel, Alès.

Les lapines témoins sont saillies à partir du sevrage qui a lieu à sept semaines. Les femelles soumises à un rythme de reproduction continue sont saillies le jour même ou le lendemain de la mise bas, à moins, ce qui est rare, qu'elles ne soient pas réceptives. Dans ce cas, des tentatives quotidiennes d'accouplement ont lieu jusqu'au succès. Leurs petits sont sevrés à I4 jours.

Tous les lapereaux sont pesés, sexés et identifiés par des encoches aux oreilles le jour de la nais. sance. Quel que soit le mode de sevrage, ils sont laissés ensemble jusqu'à 8 semaines. 


\section{Technique de sevrage}

Le sevrage est pratiqué $x_{4} \pm \mathrm{x}$ jours après la mise bas, en fin d'après-midi de manière à ce que les petits soient à jeun; en effet, selon ZARRow et al. (I965), les lapines n'allaitent qu'une fois par jour en fin de nuit ou au début de la matinée.

Aucun soin particulier n'est donné à la mère dont le tarissement se réalise sans complications.

Les lapereaux sont pris un à un, on leur frotte le museau avec de la poudre de lait, puis, après qu'ils se soient léchés, on leur trempe la bouche dans l'eau avant de les replacer dans leur nid.

Cette opération est répétée le lendemain matin et éventuellement le lendemain soir si les petits n'ont pas pris l'habitude de manger et de boire spontanément. Si l'adaptation semble lente, on peut aussi étaler un lit de foin entre la boîte à nid, l'augette et l'abreuvoir et y répandre un peu de poudre de lait.

Les aliments distribués (tabl. I) sont du lait en poudre (1), de l'eau et un granulé, le même que celui distribué aux mères, présenté sous forme de granules de $10 \mathrm{~mm}$ de long sur $5 \mathrm{~mm}$ de diamètre.

Le lait en poudre est mélangé au granulé, au moins au départ, afin que les lapins, qui initialement préfèrent le lait, s'habituent à consommer également les granulés mis à leur disposition. L'eau est distribuée dans des abreuvoirs à niveau constant.

I.e plan de rationnement adopté au cours de cet essai était le suivant:

\begin{tabular}{|c|c|c|c|}
\hline - & Lait en poudre & Granulé & Eau \\
\hline $\begin{array}{l}3^{\mathrm{e}} \text { à } 6^{\mathrm{e}} \text { semaine } \ldots . \\
7^{\mathrm{e}} \text { à } 8^{\mathrm{e}} \text { semaine } \ldots .\end{array}$ & $10 \mathrm{~g} / \mathrm{j} /$ tête & $\begin{array}{l}\text { à volonté } \\
\text { à volonté }\end{array}$ & $\begin{array}{l}\text { à volonté } \\
\text { à volonté }\end{array}$ \\
\hline
\end{tabular}

La consommation des ro g de lait était effective dès le second jour suivant le sevrage. Les quantités de granulé et d'eau consommés jusqu'à huit semaines ont été estimées par différence entre les quantités distribuées et refusées. Il n'a pas été tenu compte du gaspillage dans le calcul des quantités refusées mais celui-ci était négligeable jusqu'à six semaines, inférieur à Io p. Ioo des quantités consommées entre six et huit semaines.

\section{RÉSULTATS}

\section{a) Croissance}

Sur la figure I sont reportées les courbes de croissance des lapins appartenant à $\mathrm{I} 7$ portées témoins (IO7 individus) sevrées à sept semaines et à 24 portées ( 178 individus) sevrées à quatorze jours.

La croissance des lapins sevrés, analogue à celle des témoins au cours de la troisième semaine, est relativement plus lente pendant les trois semaines suivantes, mais devient plus rapide au cours des septième et huitième semaines. Les poids moyens à huit semaines ne diffèrent pas significativement d'un lot à l'autre et sont très voisins à neuf semaines.

\section{b) Consommation d'aliments}

Les quantités d'aliments consommées par les lapins sevrés à quatorze jours figurent au tableau 2 .

(1) “France-Lait " ̀̀ 26 p. Ioo de M. G. 
La consommation moyenne de matière sèche des lapins sevrés est considérable ; dès la première semaine de sevrage elle s'élève à $6,6 \mathrm{p}$. Ioo du poids vif par jour, atteint Io,4 p. Ioo à quatre et cinq semaines puis décroît progressivement pour être de 8,5 p. Ioo au cours de la huitième semaine.

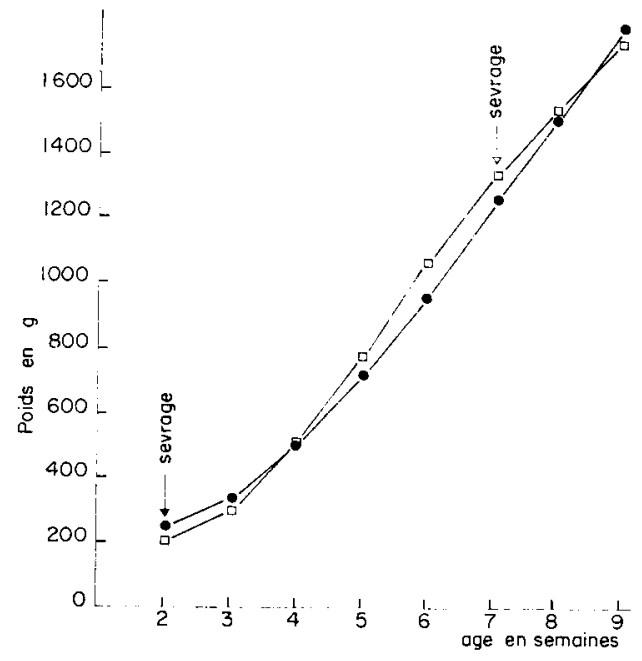

FIG. I. - Croissance comparée de lapins serrés à 2 semaines el à 7 semaines Lapins sevrés à 2 semaines.

Lapins sevrés à 7 semaines

TABLEAU 2

Consommation des lapins sevrés it deux semaines

\begin{tabular}{|c|c|c|c|c|c|c|}
\hline Semaines & $3^{\mathrm{e}}$ & $t^{e}$ & $5^{\mathrm{e}}$ & $6^{\mathrm{e}}$ & $7 \dot{e}$ & $8^{e}$ \\
\hline Nombre de portées ...... & 16 & 16 & 16 & 16 & 16 & 16 \\
\hline Nombre de lapereaux.... & 112 & 105 & 100 & 99 & 97 & 97 \\
\hline Consom. de granulés $(\mathrm{g} / \mathrm{j})$. & 9,1 & 31,3 & 60,3 & 86,9 & 112,7 & $1 \geq 6$ \\
\hline Consommation de lait $(\mathrm{g} / \mathrm{j})$ & 10 & 10 & 10 & 10 & & \\
\hline Consommation d'eau $(\mathrm{g} / \mathrm{j})$. & 31 & 63 & 97 & 128 & 167 & 192 \\
\hline $\begin{array}{c}\text { Consom. de } \text { IS (g/kg de } \\
\text { poids vif) } \ldots \ldots \ldots \ldots\end{array}$ & $i f ;$ & $10_{t}^{\prime}$ & for & 98 & 93 & 85 \\
\hline $\left.\begin{array}{l}\text { Consommation } \\
\text { d'aliments } \\
\text { par } \mathrm{kg} \text { de gain } \\
\text { de poids }\end{array}\right\}$ granule $\ldots$. & $\begin{array}{l}0,6 \\
0,7\end{array}$ & $\begin{array}{l}1,5 \\
0,5\end{array}$ & $\begin{array}{l}1,8 \\
0,3\end{array}$ & $\begin{array}{l}2,6 \\
0,3\end{array}$ & $\geq, 9$ & 3,7 \\
\hline
\end{tabular}

Les consommations d'eau et de granulé augmentent de façon à peu près linéaire jusqu'à sept semaines, plus lentement ensuite; elles s'élèvent respectivement à I92 et I26 g par jour à huit semaines. 
Le rapport eau/matière sèche augmente régulièrement, passant de $I, 4$ à trois semaines à $I, 6$ à huit semaines, il est inférieur aux valeurs que nous avons enregistrées chez des animaux âgés de neuf à vingt six semaines (PRUD'HON, Ig67) qui étaient toujours très voisines de deux.

La consommation d'aliments par kg de gain de poids des lapereaux sevrés précocement ne cesse de s'élever entre la troisième et la huitième semaine. Pour 1'ensemble de la période $2-8$ semaines, elle est en moyenne de $2,4 \mathrm{~kg}$ de granulé et $0,2 \mathrm{~kg}$ de lait.

\section{c) État de santé - Mortalité}

La mortalité des lapins sevrés à quatorze jours n'est pas globalement plus élevée que celle des lapins contemporains laissés avec leur mère : les taux respectifs de mortalité sont :

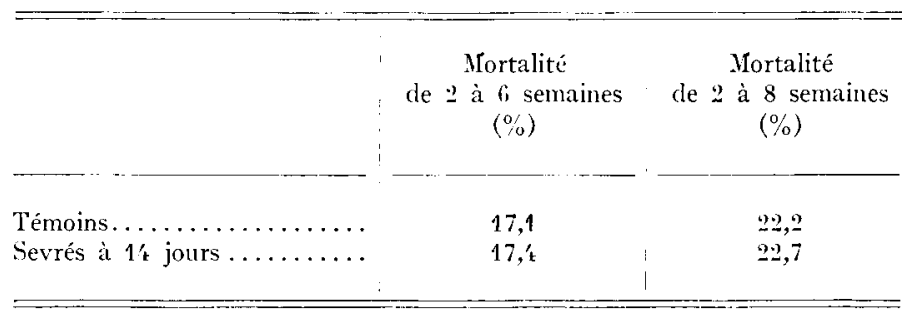

Il semble que les lapins les plus légers (moins de $200 \mathrm{~g}$ à deux semaines) soient plus fragiles lorsqu'ils sont sevrés à deux semaines que lorsqu'ils restent avec leur mère jusqu'à 7 semaines; les taux de mortalité de 2 à 8 semaines s'élèvent respectivement à $29,3 \mathrm{p}$. Ioo et $24,4 \mathrm{p}$. Ioo, la différence n'étant pas statistiquement significative. La fréquence des diarrhées, mortelles ou non, paraît malgré tout plus grande

\section{TABI,EAU 3}

Performances des lapines saillies moins de deux jours après mise bas et saillies normalement

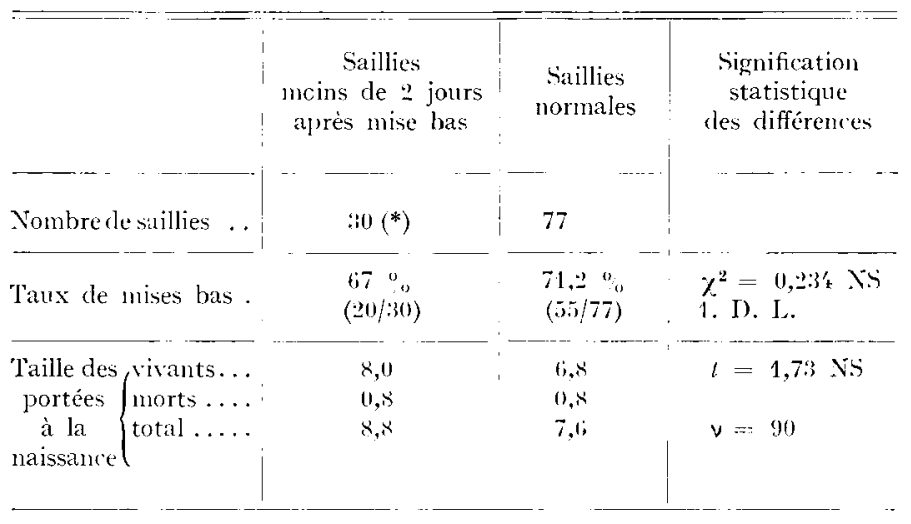

(*) Ces saillies ont été réalisées d'une part (1't (as), sur des femelles sou. mises antérieurement à un rythme normal de reproduction. d'autre part ( 16 cas), sur des femelles ayant eu deux ou trois mises bas espacces les unes cles autres de 33 a : 36 jours. 
pour les lapereaux appartenant à cette classe. La mortalité des lapins plus lourds (plus de $25^{\circ} \mathrm{g}$ à deux semaines) est faible dans les deux lots, respectivement I2,5 et I 2 p. Ioo chez les sevrés et chez les témoins.

\section{d) Reproduction continue des mères}

A l'exception de deux femelles en cstrus quinze jours seulement après la mise bas, toutes les lapines présentées au mâle dans un délai de 24 à 48 heures après parturition ont accepté l'accouplement.

Il apparaît au tableau 3 que ni la fertilité, ni la taille des portées à la naissance n'ont été diminuées significativement par la pratique d'un tel type de reproduction, mais nous manquons encore de recul pour juger des effets à échéance d'un tel rythme de mises bas.

\section{DISCUSSION ET CONCIUSION}

Ces premiers résultats ont mis en lumière plusieurs points qui semblent particulièrement intéressants.

Tout d'abord il faut souligner la rapidité d'adaptation des jeunes lapereaux à la consommation exclusive d'aliments secs : dès la première semaine de sevrage ils sont capables d'ingérer, chaque jour, une quantité de matière sèche dépassant $6 \mathrm{p}$. Ioo de leur poids et ni l'âge, ni le poids ne semblent constituer un handicap à cette adaptation puisqu'il nous a été possible de sevrer une portée âgée de dix jours 'seulement et que des lapereaux pesant I $20 \mathrm{~g}$ ont pu avoir un développement normal, la croissance record, $42 \mathrm{~g}$ par jour entre deux et six semaines ayant été réalisée par un lapin pesant I44 g au moment du sevrage. Toutefois, en règle générale, la vitesse de croissance augmente avec le poids au sevrage $(27 \mathrm{~g}$ par jour entre deux et huit semaines pour les animaux de moins de $200 \mathrm{~g}, 34,5 \mathrm{~g}$ par jour pour ceux de plus de $300 \mathrm{~g}$ ) ; le coefficient de corrélation entre le poids à deux semaines et le poids à huit semaines est : $r=0,509$ ( $\mathrm{P}<0,0 \mathrm{I})$. De plus, nous 1'avons vu, la mortalité est beaucoup plus faible chez les animaux les plus lourds.

Cependant, la comparaison des croissances d'animaux élevés traditionnellement et d'animaux sevrés précocement montre que la croissance de ces derniers est freinée de trois à six semaines, une croissance compensatrice leur permettant de rattraper leur retard de la septième à la neuvième semaine ; ceci reflète sans doute l'insuffisance qualitative ou quantitative des rations distribuées. Le lait de lapine, en effet, est extrêmement riche en matières grasses et matières azotées ainsi que le montre la revue bibliographique de LEBAs (I965) et les travaux de CoA'tes et al. (I964), DaviES et al. (I964) :

\begin{tabular}{|c|c|c|c|}
\hline $\begin{array}{l}\text { Composition du lait } \\
\text { de lapine }\end{array}$ & LEBAS, 1965 & $\begin{array}{c}\text { COATES } \\
\text { et } a l ., 1965\end{array}$ & $\begin{array}{c}\text { DAVIES } \\
\text { et } a l ., 196^{\prime}\end{array}$ \\
\hline & $(\%)$ & $(\%)$ & $(\%)$ \\
\hline Teneur en matière sèche. & 30,5 à 45,7 & $25,7 \dot{a} \quad 36,0$ & 32,8 \\
\hline Matières grasses $\% /$ MS... & $3{ }^{\prime}, 3$ à 55,3 & 31,8 à 5 & 45 \\
\hline Matières azotées \%/MS . & 33,1 à 50,9 & 42,6 ถ̀ 46,3 & 41 \\
\hline
\end{tabular}


Nous avons constaté, par contrôle laitier indirect, qu'un lapereau consommait en moyenne $35 \mathrm{~g}$ de lait par jour à l'âge de 3 semaines, soit 9 à $\mathrm{I} 6 \mathrm{~g}$ de matière sèche, 3,5 à $7,5 \mathrm{~g}$ de matières azotées et 3,4 à $8,6 \mathrm{~g}$ de matières grasses par jour ; à cet âge, notre ration leur apporte $3,4 \mathrm{~g}$ de matières grasses et $7 \mathrm{~g}$ de matières azotées dont $2,6 \mathrm{~g}$ seulement proviennent du lait.

On peut donc se demander si les lapereaux sevrés précocement sont capables de compenser l'insuffisance de matières grasses de la ration par l'utilisation d'autres sourcess d'énergie?

On peut également se demander s'ils ne souffrent pas temporairement d'une carence partielle en acides aminés indispensables? En effet, si les besoins azotés de croissance des lapins sont mal connus (LEBAS, I965), les travaux de WoOLEY (I954), Hove et HeRNDON (I957) et CASADY (I96I) permettent de penser que la croissance des jeunes lapereaux dépend étroitement de la qualité des protéines de leur ration.

Il faut souligner également l'aptitude étonnante de la plupart des lapines à se laisser saillir immédiatement après la mise bas et à avoir une gestation normale à la suite de cette saillie.

En I925, Hammond et Marshal, avaient signalé que la lapine peut être saillie et ovuler après la mise bas, mais selon ces auteurs, les embryons mouraient au stade blastocyte si la lapine allaitait une portée de plus de trois à quatre petits. CASADY (I965), a réussi à obtenir une moyenne de $I 7$ portées en 26 mois chez trois lapines dont il abattait systématiquement les petits à la naissance. DON Guthrie (I966) a mis au point un élevage intensif avec saillie à partir de dix jours et sevrage à 24 jours sans inconvénients majeurs pour les lapines.

Les résultats que nous avons obtenus permettent de penser qu'une saillie post partum et un allaitement de quinze jours sont sans effets néfastes sur le déroulement normal d'une gestation ni sur le nombre des petits qui naissent.

En résumé : le sevrage précoce des lapereaux avec administration d'une alimentation sèche est possible.

La croissance des lapins ainsi sevrés est peu différente de celle des témoins et la mortalité n'est pas significativement supérieure à celle des lapereaux qui tettent.

Dans la mesure où la santé et la fertilité des lapines ne seront pas altérées par des gestations successives, il est permis d'envisager une augmentation spectaculaire de leur productivité par la pratique des saillies le jour de la mise bas et du sevrage précoce des lapereaux.

\section{Reçu pour publication en janvier 1968.}

\section{SUMMARY}

EARLY WEANING OF YOUNG RABBITS AND REPRODUCTION OF DOES

The performances of 178 rabbits from 24 litters weaned at 2 weeks of age were compared with those of 107 rabbits from 17 litters suckled for 7 weeks.

The weaned rabbits were given with :

- Io g of milk powder at 26 per cent fat level until 6 weeks ;

- a granulated at 20.5 per cent nitrogen content, available ad libitum;

- water provided continuously. 
Their growth rate from 2 to 8 weeks ( $30 \mathrm{~g}$ per day) and their weight at 8 weeks ( $1504 \mathrm{~g}$ ) did not significantly differ from the controls. The growth rate of the controls was greater from 3 to 6 weeks of age, but the difference was balanced by a higher rate of gain of the weaned rabbits from 7 to 9 weeks.

Mortality rates from 2 to 8 weeks did not significantly differ : 22.7 for the weaned rabbits versus 22.2 for the controls, though the animals which were heavier at weaning seemed to be less subject to diarrhea than controls of the same weight.

The does of early-weaned litters were mated again on the day of parturition ; 67 per cent became pregnant. The average following litter was 8.8 rabbits. Neither birth rate nor size of litters at birth differed significantly from those of control females mated during the same season.

Our results point out the possible use of weaning at 14 days of age together with mating immediately after parturition to improve reproductive performances.

\section{RÉFÉRENCES BIBLIOGRAPHIQUES}

Casaiy R. B., Damon R. A., Suttor A. E., ig6I. Effect of supplementary lysine and methionine on enteris mortality, growth and feed efficiency in young rabbits. J. Nutr., 74, 120.

Casaly R. B., 1965. Onze portées par all. Vos lapizhs, 85, it-zo.

Contes M. E., Gregory M. E., Trompson S. Y., I964. The composition of rabbits milk. Brit. J. Nutr., 18, $583-587$.

Davies J. S., Windowson F. M., McCance R. A., ig6.4. The intake of milk and retention of its constituents while the newborn rabbit doubles its weight. Brit. J. Nitr., 18, 385-392.

Guturie D., 1960. Creep feeding, sevrage précoce et accélération du rythme reproductif. Vos lafins, 86, $18-24$.

Hammoni) J., Marshall F. M. A., 1925. Reproduction in the rabbit. Edimburgh 1925. (ite par AsDell S. A., 1946. Palterns of mammalian reproduction. Constock New York.

Hove E. L., Heridon J. F., 1957. Growth of rabbits on purified diets. J. Nutr., 63, 193.

LEBAS F., I 965 . J'alinentation du lapin (communication personnelle).

Pruv'ron M., I967. L'appétit du lapin alimenté à sec. Bull. Téh. Int., mai 1067, 383-398.

Soubielle V., Ig66. Un élevage de sélertion Fauve de Bourgogne. Tribune du nonde rural, oct. 1966, 6-8.

Wooley I. G., 1954. Growth of three to four week old rabbits fed purified and stock rations. J. Nulr. 29, rgr. Cité par Lemas F., L'alimentation du lapin (communication persomelle).

Zarrow M. X., Denenberg V. M., ANuerson C. 0 , ig65. Rabbit frequency of suckling in the pup. Science, 150, I $835-36$. 